\title{
La percepción del conflicto escolar en tres comunidades de aprendizaje
}

The Perception of School Conflict in Three Learning Communities

La perception du conflit scolaire de trois communautés d'apprentissage

A percepção do conflito escolar em três comunidades de aprendizagem

Fecha de recepción: 10 DE ENERO DE 2017/Fecha de aceptación: 7 DE NOVIEMBRE DE 2017/Fecha de disponibilidad en línea: 15 DE ABRIL DE 2018 Encuentre este artículo en http://magisinvestigacioneducacion.javeriana.edu.co/

Escrito por María Teresa Vizcarra-Morales Universidad del País Vasco/EusKal Herriko Unibertsitatea Lejona, España mariate.bizkarra@ehu.eus

ItZiar Rekalde-Rodríguez Universidad del País Vasco/Euskal Herriko Unibertsitatea
Lejona, España itziar.rekalde@ehu.eus

Ana María Macazaga-López Universidad del País Vasco/Euskal Herriko Unibertsitatea Lejona, España ana.macazaga@ehu.eus

\begin{abstract}
Resumen
Esta investigación analiza la percepción del conflicto en tres comunidades de aprendizaje para explorar el significado que atribuyen al término conflicto y averiguar con qué escenarios asocian su aparición. La metodología reside en la perspectiva comunicativa crítica y las técnicas han sido los relatos y las entrevistas. Los resultados indican que el conflicto se considera un hecho natural que en ocasiones deriva en comportamientos agresivos y que hay espacios que facilitan su aparición. Las conclusiones reflejan la necesidad de contemplar la dinámica procesual del conflicto y ofrecer contextos educativos en los que experimentar y aprender a gestionar las emociones.
\end{abstract}

\section{Palabras clave}

Conflicto; educación primaria; comunidades de aprendizaje; investigación comunicativa crítica; diálogo

Para citar este artículo / To cite this article / Pour citer cet article / Para citar este artigo

Vizcarra-Morales, María Teresa; Rekalde-Rodríguez, Itziar \& Macazaga-López, Ana María (2018). La percepción del conflicto escolar en tres comunidades de aprendizaje. magis, Revista Internacional de Investigación en Educación, 10 (21), 95-108. doi: 10.11144/Javeriana.m10-21.pceca 


\section{Keywords}

Conflict; primary education; learning communities; critical communicative research; dialogue

\section{Mots clés}

Conflit ; éducation primaire ; communautés d'apprentissage; recherche communicative critique ; dialogue

\section{Abstract}

This research analyzes the perception of conflict in three learning communities to explore the meaning they attribute to the term conflict and to find out in which scenarios they associate their emergence. The methodology follows a critical communicative perspective and the techniques are stories and interviews. Results indicate that conflict is considered a natural fact that sometimes results in aggressive behavior and that there are spaces that facilitate its emergence. We concluded that there is a need to contemplate the processual dynamics of conflict and offer educational contexts in which to experiment and learn to manage people's emotions.

\section{Résumé}

Cette recherche analyse la perception de trois communautés d'apprentissage sur la signification attribuée au terme conflit, on enquête sur quels scénarios les communautés associent son apparition. La méthodologie se base sur la perspective communicative critique et les techniques ont été les récits et les entretiens. Les résultats indiquent que le conflit se considère comme un fait naturel qui parfois dérive en comportements agressifs et qu'il y a des espaces qui facilitent son apparition. Les conclusions montrent le besoin de contempler la dynamique processuelle du conflit et fournir les contextes éducatifs dans lesquels expérimenter et apprendre à gérer les émotions.

\section{Palavras-chave}

Conflito; educação primária; comunidades de aprendizagem; pesquisa comunicativa crítica; diálogo

\section{Resumo}

Esta pesquisa analisa a percepção do conflito em três comunidades de aprendizagem para explorar o significado que atribuem ao termo conflito e averiguar com que cenários associam seu aparecimento. A metodologia reside na perspectiva comunicativa crítica e as técnicas têm sido os relatos e as entrevistas. Os resultados indicam que o conflito se considera um fato natural que em ocasiões deriva em comportamentos agressivos e que há espaços que facilitam seu aparecimento. As conclusões refletem a necessidade de contemplar a dinâmica processual do conflito e oferecer contextos educativos nos quais se experimente e se aprenda a administrar as emoções. 


\section{Introducción}

El conflicto es un tema que no pasa de moda y que está presente en las instituciones, en general, y en la escuela, en particular. La escuela se caracteriza por convivir cotidianamente con diferentes tipos de conflicto. Pero ¿qué significado tiene este concepto para los participantes en el contexto escolar? La orientación positiva del conflicto y los planteamientos del aprendizaje dialógico implementados en las comunidades de aprendizaje (CCAA) nos ayudan a descubrir cómo se puede abordar el conflicto desde su detección al implicar a la comunidad educativa para ir construyendo una convivencia educativa intercultural e inclusiva en el centro (Flecha-García \& García-Yeste, 2007; Leiva-Olivencia \& Escarbajal-Frutos, 2011; Pérez-Galán, 2009; Vargas \& Flecha, 2000). Pero, hasta el momento, no se ha hecho hincapié en cómo percibe el conflicto la comunidad educativa, qué significado le da y con qué otros conceptos lo vincula.

Esta preocupación no es baladí, dado que la percepción que tienen los participantes en CCAA puede aportar conocimiento sobre el tema que ayude a mejorar las actuaciones de éxito, orientadas a hacer del conflicto una herramienta pedagógica al servicio del cambio y la transformación tanto individual como grupal.

\section{Aproximación conceptual al conflicto como posibilidad de cambio y transformación}

La percepción del conflicto varía según el paradigma educativo desde el que se construye. Desde un enfoque tecnocrático, está cargado de una connotación predominantemente negativa por interpretarse como un elemento perturbador de la convivencia y del proceso de enseñanzaaprendizaje. Mientras que, desde una perspectiva crítica, es percibido como un elemento potencialmente positivo porque aporta la posibilidad de cambio tanto personal como social y ayuda a establecer identidades individuales y grupales (Jares, 2001; Leiva-Olivencia, 2009; Pineda-Alfonso, 2011). Desde esta perspectiva no solo se ve como posibilidad de cambio sino que es considerado un rasgo natural e inherente a las relaciones sociales, que emerge de forma inevitable en el proceso de confrontación y defensa de las propias posiciones, intereses y necesidades; su ausencia es interpretada como adormecimiento, apatía e indiferencia que aleja a las personas y a la comunidad escolar de la posibilidad de cambio y transformación creativa.

La idea de poner la confrontación al servicio de la transformación creativa coincide con la perspectiva de la filosofía de la paz en la medida en que esta considera útil y necesario para la convivencia que las personas aprendan a confrontar y tomarse en cuenta unas a otras de forma respetuosa y pacífica (París-Albert, 2005; París-Albert \& Martínez-Guzmán, 2010). Por tanto, desde la perspectiva crítica, el abordaje pedagógico del conflicto y la confrontación se presenta como requisito para la transformación personal y social. El reto o desafío es realizarlo de forma respetuosa y pacífica.

En este punto, resulta oportuno señalar la confusión que hay entre agresividad y violencia, así como entre violencia y conflicto. La explicación aportada desde la bioenergética distingue entre dos tipos de agresividad (Lowen \& Lowen, 2005), la agresividad nociva orientada a destruir o causar un mal a otra persona, a sí mismo, o al entorno, y una agresividad nutricia o energía de vida necesaria para enfrentar de forma asertiva las situaciones de conflicto que surgen en las relaciones humanas. El valor nutricio de la agresividad se refiere a las dos funciones complementarias que desempeña:
Descripción del artículo | Article description | Description de l'article | Artigo descrição

Este artículo de investigación, derivado del proyecto "Juega, dialoga y resuelve: Diseño de un programa específico para Comunidades de Aprendizaje", analiza cómo es percibido el conflicto y en qué espacios se genera con mayor facilidad, de cara a conocer las claves sobre las que intervenir en un futuro. Las comunidades de aprendizaje desean abordar el conflicto desde una perspectiva dialógica y comprensiva. 
"Por una parte constituye una fuerza activa para el propio desarrollo, por otra, es un instrumento para defender la propia identidad de todo aquello que la amenaza" (Bonino, 1987, p. 7).

Resolver los conflictos de forma positiva está ligado a poner la agresividad nutricia al servicio de la resolución pacífica y constructiva del conflicto de manera que nadie pierda, y se opone al carácter negativo y destructivo de la agresividad nociva que se hace presente en la gestión del conflicto mediante actos de agresión (Cascón-Soriano, 2000; Jares, 2001; Martínez-Guzmán, 2005; París-Albert, 2005). Sin embargo, para avanzar en el cambio y la transformación creativa del conflicto, no basta con hacer uso de la energía nutricia; hace falta, además, ahondar en las causas que alimentan el conflicto. Es decir, conlleva la necesidad de adoptar una perspectiva procesual que reduzca el conflicto a la categoría de manifestación observable. Por el contrario, implica diferenciar que una cosa es el conflicto y las causas que lo provocan, y otra, las manifestaciones y las actitudes que utilizan los protagonistas para afrontarlos. Adoptar esta posición supone prestar atención a los síntomas para indagar sobre el origen del verdadero problema que subyace a todo conflicto (Cascón-Soriano, 2000; Jares, 2001; Martínez-Guzmán, 2005).

Así, en el contexto de aula, el profesorado que adopta esta perspectiva procesual, al tiempo que mejora sus prácticas, aprende sobre sí mismo (Bolívar-Botía, 2000; Naranjo, 2014; Palomero-Fernández, 2010; Perrenoud, 2004). Por ejemplo, un profesor de primaria que está impartiendo una 'dilatada' explicación, observa que algunos niños están hablando, y no prestan atención a sus consignas. En lugar de adoptar, de forma inmediata, medidas disciplinarias se tomará un tiempo para comprender qué puede estar motivando la falta de atención y de motivación. Se preguntará por las necesidades no cubiertas o los sentimientos de insatisfacción que puedan haber inducido al alumnado a comportarse así. Se preguntará por las razones que le han llevado a esperar que el alumnado atendiera a su propuesta y se mostrará motivado por ella. Plantearse este tipo de interrogantes y reflexionar sobre estas cuestiones le posibilitará no solo cuestionarse sus prácticas y mejorarlas, sino también aprender sobre sí mismo y su alumnado.

Adentrarse en las causas del conflicto es tarea complicada y priorizar unas $u$ otras dependerá de la teoría y disciplina desde la que se teoriza (Jares, 2001; Vinyamata, 2003). Los factores que contribuyen a la aparición del conflicto en el ámbito escolar están habitualmente relacionados con cuestiones de clima y contexto organizativo o metodológico y con cuestiones relativas al ámbito de las relaciones interpersonales y socioemocionales (De la Caba-Collado, 1997). En contextos interculturales, resulta complejo determinar las causas, ya que son producidas por una multicausalidad de significados (Colectivo Amani, Aguilera-Reija, Gómez-Lara, Morollón-Pardo \& De Vicente-Abad, 2009; Leiva-Olivencia, 2009; Ortega, Mínguez \& Saura, 2003).

Según Rosa Cobo-Bedia (2006), una parte significativa de los conflictos culturales que se manifiestan en estos contextos son conflictos de género que tienen dimensiones fuertemente patriarcales por recaer sobre ellos - y ellas - guiones de vida diferenciados; entre los elementos en disputa están el uso del velo islámico, las prácticas deportivas... Otros estudios sitúan en el origen del conflicto la no satisfacción de necesidades humanas básicas, como los sentimientos de pertenencia, aceptación y respeto por parte del grupo de iguales y de las personas adultas de referencia (Llamas-Toledano, 2003). Numerosas voces reclaman la importancia de prestar atención al ámbito emocional, al compartir la idea de que las 
emociones que acompañan a un conflicto — como miedo, ira, desconfianza, suspicacia, desprecio, rechazo, resentimiento, entre otras - responden a necesidades no satisfechas y deben poder ser expresadas, escuchadas y atendidas para evitar que deriven en comportamientos violentos (Díaz-Aguado, 2006; Martínez-Guzmán, 2005; París-Albert, 2005). De hecho, el estudio de Catherine Blaya y Éric Debarbieux (2011) en los colegios franceses de primaria puso de manifiesto que el $10 \%$ del alumnado sufre la victimización repetida que les produce un sentimiento de inseguridad y afecta su percepción del clima escolar.

El patio es referido por la literatura como el contexto escolar en el que se producen más conflictos, donde quienes son más fuertes abusan de quienes son más débiles (Ruiz, Riuró \& Tesouro, 2015; SalinasSalazar, Posada-Giraldo \& Isaza-Mesa, 2002). La mayor parte de las peleas que se producen en la escuela se da entre niños y niñas, o entre niños solos, siendo estas últimas las más frecuentes. La mayor presencia de comportamientos agresivos entre los chicos se explica por su dificultad para comunicar y expresar sus emociones en situaciones de tensión o estrés y por su mayor predisposición fisiológica a la acción (Trianes-Torres, 2000). Entre las áreas curriculares, la educación física es identificada como la más propensa a la aparición de conflictos, debido a que los cuerpos interaccionan más tiempo y más intensamente en las actividades que se proponen (Martín-Hocajo \& Ríos-González, 2014). Los conflictos habituales están ligados a la práctica de actividades competitivas en las que se prioriza la búsqueda de la victoria, lo que da lugar a discrepancias por diferentes niveles de habilidad o personalidad, por la cultura o el género, por el uso del material y el espacio (Ortí-Ferreres, 2003). Prueba del valor pedagógico de esta área curricular, como laboratorio en el cual experimentar la propia emocionalidad, tomar conciencia de ella y aprender a manejarla, son las numerosas publicaciones que se han realizado en los últimos años (Antolín de la Fuente, Martín-Pérez \& Barba-Martín, 2012; Hellison, 2010; Lavega, Filella, Lagardera, Mateu \& Ochoa, 2013; Macazaga-López, Rekalde-Rodríguez \& Vizcarra-Morales, 2013; Monjas-Casares \& GonzálezMoreno, 2000; Sáez de Ocáriz, Lavega \& March, 2013; Vizcarra-Morales, Macazaga-López \& RekaldeRodríguez, 2013).

No obstante, el tipo de escuela marca, así mismo, el enfoque mediante el cual abordar las situaciones conflictivas. En el caso de las CCAA se comparte una visión del conflicto como oportunidad para construir convivencia (Elboj-Saso \& Niemelä, 2010; Gómez, Latorre, Sánchez \& Flecha, 2006). Su actuación se centra en cómo resolver el conflicto una vez detectado. En coherencia con los principios del aprendizaje dialógico que sustenta sus prácticas, se orientan a capacitar a la comunidad para resolver los problemas por medio del diálogo (Elboj-Saso \& Niemelä, 2010). El modelo que adoptan es el comunitario que se basa en la prevención y resolución de conflictos, que como su propio nombre indica implica trabajar con toda la comunidad (Flecha-Fernández de Sanmamed, Melgar, Oliver \& Pulido, 2010; Vizcarra-Morales, Macazaga-López \& Rekalde-Rodríguez, 2016). Entre las acciones que vienen planteando destacan la socialización preventiva en la violencia de género (Elboj-Saso, 2011), y la asignatura de educación física como escenario privilegiado para trabajar la transformación creativa del conflicto (Monzonís-Martínez \& Capllonch-Bujosa, 2014).

\section{Metodología}

Objetivos: esta investigación ha explorado la percepción que del conflicto tienen las personas implicadas (escolares, familiares, voluntariado y profesorado) en tres CCAA, mediante un análisis profundo de distintas situaciones conflictivas, para aportar conocimiento que contribuya a mejorar las actuaciones de éxito. En este sentido, los objetivos han sido explorar el significado que los participantes atribuyen al término conflicto y averiguar con qué escenarios asocian su aparición.

Posicionamiento metodológico: desde una orientación positiva del conflicto y teniendo en cuenta el contexto de CCAA en el que se enmarca la investigación, la perspectiva metodológica está asentada en los presupuestos de la metodología comunicativa crítica (Gómez, Latorre, Sánchez \& Flecha, 2006; Gómez, Puigvert \& Flecha, 2011). Desde esta perspectiva, la realidad social se entiende como una construcción humana cuyos significados son construidos de forma comunicativa por medio de la interacción entre las personas, y donde la estrategia de construcción del conocimiento reside en el diálogo. Esta metodología permite la sistematización, valoración y visibilización de la palabra de personas que, desde el espacio cotidiano, problematizan la realidad con una concienciación emancipadora para que deriven en prácticas transformadoras de su entorno (Flecha-Fernández de Sanmamed, Melgar, Oliver \& Pulido, 2010).

Técnicas de recogida de información: siguiendo esta perspectiva, se utilizan diferentes técnicas de naturaleza cualitativa y en clave dialógica, como los relatos comunicativos y la entrevista comunicativa (Gómez, Latorre, Sánchez \& Flecha, 2006). Estas técnicas han permitido, desde la polifonía que supone interactuar con diferentes colectivos, indagar sobre la percepción que tienen del conflicto; qué supone, en qué situaciones se origina... Todo ello para 
identificar el enfoque que hay detrás de estas percepciones y perfilar pautas de actuación que ayuden a poner el conflicto al servicio de los procesos de transformación personal y social. Las relaciones entre las personas entrevistadas y las investigadoras han buscado la horizontalidad para diluir las relaciones de poder y beneficiar la comunicación (Flick, 2014; Valles-Martínez, 2003).

Participantes: en el estudio han participado 3 centros pertenecientes a CCAA; dos de ellos públicos (C1 y C2) y el tercero concertado (C3). Se contó con una persona participante por colectivo y centro. Se realizaron 3 relatos comunicativos ( 2 alumnos y 1 alumna de $5^{\circ}$ y $6^{\circ}$ de educación primaria), y 9 entrevistas comunicativas, con 1 profesor y 2 profesoras, 3 madres y 3 voluntarias.

Procedimiento de análisis, dimensiones y categorías: el procedimiento analítico ha sido inductivo-deductivo en el que las dimensiones del sistema categorial (tabla 1) fueron las preguntas planteadas en la investigación (¿Qué tipo de agresividad percibe la comunidad? ¿El modo de organizar el espacio o las actividades es facilitador de los conflictos?). El proceso de agrupación de los datos en categorías requirió determinar qué información era relevante para los objetivos de la investigación; por ello, se unificaron los criterios de pertenencia a las categorías establecidas.

El sistema categorial resultante se muestra en la tabla 1.

Tabla 1

Sistema categorial

\begin{tabular}{l|l}
\hline Dimensiones & Categorías \\
\hline $\begin{array}{l}\text { Hacia la comprensión } \\
\text { del conflicto }\end{array}$ & $\begin{array}{l}\text { Agresividad nutricia: el conflicto como discusión, } \\
\text { roces y pequeñas faltas es inevitable en } \\
\text { situaciones de convivencia (transformadora). }\end{array}$ \\
\cline { 2 - 2 } & $\begin{array}{l}\text { Agresividad nociva: el conflicto como agresión } \\
\text { asociada a insultos y peleas (exclusora). }\end{array}$ \\
\hline \multirow{2}{*}{$\begin{array}{l}\text { de conflicto favorecedores } \\
\text { Espacios libres: situaciones en las que el } \\
\text { alumnado se siente libre para hacer, expresar y } \\
\text { moverse (transformadora). }\end{array}$} \\
\cline { 2 - 2 } & $\begin{array}{l}\text { Espacios de competición: situaciones } \\
\text { competitivas de ganar y perder (exclusora). }\end{array}$ \\
\hline \hline
\end{tabular}

Fuente: elaboración propia

La información recogida se ha ordenado para su análisis e interpretación haciendo uso del programa NVIVO11. Para cumplir los requisitos de confidencialidad, la procedencia de cada voz ha sido identificada con códigos que responden a los siguientes criterios: centro de pertenencia $(C 1, C 2$, $C 3)$, técnica comunicativa: relato $(R)$, entrevista $(E) y$, por último, colectivo: alumnado $(A)$; profesorado $(P)$; familias $(F)$ y voluntariado $(V)$.

Credibilidad de la información: la credibilidad se ha conseguido mediante la triangulación de las opiniones de las personas informantes que procedían de diferentes colectivos (Denzin \& Lincoln, 2015), para crear nuevas conexiones al comparar y contrastar los datos desde diferentes ángulos y perspectivas (Elliott, 2010).

\section{Resultados}

Haciendo uso del sistema categorial obtenido durante el proceso de análisis, se presentan a continuación los resultados del estudio. 


\section{Hacia la comprensión del conflicto}

Las personas participantes expresan qué entienden por conflicto recurriendo al uso de numerosos términos como discusión, roce, enfado, pelea, insulto, falta de respeto, falta de empatía... Han sido agrupados atendiendo a la mayor o menor carga de agresividad nociva referida en la descripción de la expresión utilizada.

\section{Agresividad nutricia: el conflicto como discusión, roces y pequeñas faltas} es inevitable en situaciones de convivencia

Todas las personas adultas se refieren al término discusión para expresar lo que entienden por conflicto. Familias, voluntariado y profesorado asocian discusión al resultado natural de estar en desacuerdo en algo, por tener puntos de vista o intereses distintos. Aluden a que cualquiera puede verse implicado en una discusión.

...el conflicto es normal porque todos no somos iguales y podemos opinar de una forma o de otra y chocar en algunos momentos. Eso es normal. Los conflictos van a existir siempre, son inevitables. La forma de gestionarlos es la diferencia (C2_EP)'.

Desde esta perspectiva de inevitabilidad del conflicto, familias, voluntariado y profesorado expresan que no hay razón para asociar la existencia de discusiones y desacuerdos a situaciones negativas. Coinciden en señalar que el verdadero problema para la convivencia se sitúa en la incapacidad de las personas de resolver el conflicto sin que ninguna de las partes se sienta dañada.

Yo creo que los conflictos son habituales, pero vamos a ver que todos no tienen por qué ser traumáticos desde el punto de vista de que hay distintos intereses. Si hay conflictos, no tienen por qué ser negativos, ni traumáticos, ni nada... nadie tiene por qué quedarse dolido y romper las relaciones... (C1_EV).

Desde la perspectiva de inevitabilidad del conflicto, las personas adultas utilizan las expresiones roces y pequeñas faltas para verbalizar lo que entienden por conflicto sin importancia. Y así, empujones, tropezones, golpes, choques, encontronazos... forman parte del día a día de la vida en la escuela en situaciones de juego y de contacto físico, ya que la excitación y la tensión nerviosa aumentan. El profesorado no considera graves estas situaciones, porque no aprecia que haya falta de respeto $y$, porque se resuelven pidiéndose perdón para continuar con el juego. Considera necesaria la intervención docente cuando percibe faltas de respeto y actitudes de pelea que alertan sobre la posibilidad de entrar en una espiral de agresiones verbales o físicas.

Es que es la convivencia, el roce... pero yo no creo que sea nada fuera del otro mundo y nada grave, ni nada desagradable, no son pequeñas tonterías que rozan entre ellos, ....son momentos de nervios, de tensión, que se les va un poco la mano... nada más... pero al momento están bien jugando otra vez, reconocen, se piden perdón, pero si un compañero falla y se da un grito menospreciando o insultando al que ha fallado, esto sería una falta de respeto y ese momento requeriría una intervención para solucionarlo y evitar que fuera a más (C3_EP).

1 magis no edita ni corrige las declaraciones de los entrevistados. Por eso, mantiene las marcas del lenguaje oral, los dichos, muletillas y modismos. 
De esta manera, la agresividad nutricia es un elemento transformador que ayuda en la construcción de la convivencia.

Agresividad nociva: el conflicto como agresión asociada a insultos y peleas

Cuando las personas participantes utilizan estos términos lo hacen para referirse a situaciones en las que tiene lugar una agresión que habitualmente adopta la forma de insulto o pelea física y llega a las manos. Todas las voces asocian estos comportamientos a situaciones de enfado en las que la persona que se siente ofendida o atacada, busca el modo de devolver el ataque o la ofensa, con lo cual se entra en una espiral que conduce a comportamientos violentos.

Pues si alguien te hace la puñeta y tú te enfadas y muchas veces, pues al final, se la devuelves aunque eso no lo tengas que hacer. Eso es algo que suele pasar normalmente en la escuela, suele suceder bastantes veces, y al final acaba mal (C1_RA).

Las voces más jóvenes apuntan a que la mayor parte de los conflictos que se producen en la escuela se debe al pique existente entre chicos y chicas. Las expresiones de menosprecio y los insultos entre ellos y ellas suelen ser frecuentes, aunque saben que no es la manera de resolver sus diferencias. Una niña expresa que la causa de estos conflictos se encuentra en la actitud machista de la que los chicos hacen gala, actitud de superioridad respecto a ellas, que se hace especialmente visible en las prácticas deportivas.

...el pique que más se da en clase es entre chicos y chicas, estamos muy separados. Cuando los chicos dicen algo, las chicas nos enfadamos con los chicos; y cuando las chicas decimos algo, los chicos [se enfadan] con las chicas. Y una vez yo estaba mazo enfadada, porque un niño le estaba diciendo a otro niño que las chicas tenemos menos fuerza para jugar, que si tuvieran que elegir en gimnasia, elegirían primero a los chicos y todo eso... y me parece mazo mal, porque me parece mucho machismo... hay un niño que es supermachista porque dice que las chicas somos más débiles, y cuando elige, en vez de escoger todos chicos y después todas las chicas, escoge las chicas que más fuerza tengan y luego las últimas te las regala, no sé, como si no valiesen nada... como si fueran juguetes... Que digan eso de 'te las regalo' [pone cara de asco] como si fuera un juguete, que te la doy pa' ti, pa' tu grupo (C2_RA)

Todas las personas entrevistadas consideran el insulto un elemento detonador de conflicto. El argumento más utilizado es que si hay insulto, hay falta de respeto y, por tanto, conflicto.

...un conflicto escolar también es que una persona esté insultando a otra llamándole gorda, un día y otro día... por ejemplo, es un acoso... El niño este, por ejemplo, que viene con mi hija al colegio, como es un crío que es obeso, que es gordo ino?, pues, le llama gordito... a lo mejor para ti es una gracia, pero a él le estás haciendo daño... más que si le metes un tortazo... eso es acoso y no hay derecho, habría que cortarlo (C2_EV).

Una de las voluntarias repara en la dificultad de algunos escolares para gestionar sus enfados, que ante la carencia de recursos para manejar la frustración e impotencia recurren al insulto. Las víctimas de este tipo de agresiones verbales suelen ser personas de diferente etnia o religión. 
... mira, con esto del insulto, hemos tenido hace poco un conflicto importante en el centro, a cuenta de su situación de inmigrantes, entre un niño que es de aquí, del País Vasco y otros niños que no lo son... el problema estaba en que este niño en cuanto se enfada o algo le sale mal se pone muy nervioso y no sabe cómo defenderse y se defiende insultando y atacando... (C2_EV).

El profesorado y las familias coinciden en destacar que el término gitano, utilizado con el propósito de molestar, ha cedido su lugar al término gordo-gorda poniendo de relieve el protagonismo que ha adquirido el aspecto físico como elemento de discriminación y menosprecio.

...ahora por ejemplo para insultarse se llaman 'gordo' pero ya ese insulto de gitano, vete de aquí... eso desapareció, está borrado, completamente. Es una de las cosas que, hace muchos años, cuando empezamos aquí, se usaba ese insulto, ahora se usa el de gordo con la idea de hacer daño... (C2_EP).

\section{Escenarios favorecedores de conflicto}

Hemos agrupado la información en dos categorías que responden, a su vez, a dos factores que son la vivencia de sentirse libre y la competitividad.

Espacios libres: situaciones en las que el alumnado se siente

libre para hacer, expresary moverse

Las voces nos remiten a dos tipos de situaciones. Por un lado, situaciones ligadas a la ausencia de vigilancia y control y, por otro, aquellas en las que el espacio no está restringido por sillas y mesas, y ofrecen una posibilidad de juego y contacto físico que activa reacciones impulsivas que suelen derivar en conflicto. Las personas entrevistadas se refieren al patio, al vestuario y a las clases de educación física como escenarios preferentes.

Todas las personas participantes coinciden en señalar el patio como el lugar más conflictivo por la ausencia de vigilancia y control. Una madre expresa lo peligroso que puede llegar a ser este espacio, por los abusos de poder que perpetran quienes son más fuertes contra quienes son más débiles.

... a mí el patio me parece un lugar particularmente problemático, y yo sé que los niños tienen que aprender a vivir en sociedad... pero lo del patio es indignante y hay una cosa que me ha preocupado siempre, pero que no le veo solución o no conozco. La cosa es que cuando los niños salen al patio y no hay padres, suele haber pocos profesores para una barbaridad de niños, con lo cual hay la posibilidad de que haya abusos de los más fuertes contra los más débiles, que siempre ha existido... creo yo que el germen del acoso por ahí va... lo del patio me parece fuerte, muy fuerte (C1_EF).

En este mismo sentido se sitúa la siguiente voz de una alumna.

(...) cuando estamos en el patio, los chicos están jugando al fútbol se cierran en banda, tienen sus peleas entre ellos porque es un baile de chicos... El fútbol es todo de chicos y hay problemas... con un chico que es de $A$, va el niño y le dice tú no juegas porque... no eres de mi curso... (C2_RA). 
El vestuario es otro de los lugares problemáticos ligados a la ausencia de vigilancia, porque suelen producirse menosprecios y humillaciones ligadas al aspecto físico.

Mi hijo pequeño tuvo un problema en la clase de educación física, hubo un malentendido en clase: tenían que participar todos corriendo y él no se encontraba bien, le comentó al profesor y se sentó. Entonces, los compañeros de clase empezaron a llamarle 'nenaza', que no sé qué... y, bueno, quedó ahí la cosa, pero luego en el vestuario la cosa fue a más, subió de tono, se metieron con él... con su físico... y salió mal, salió llorando (C3_EF).

En lo que se refiere a la educación física una profesora argumenta que esta asignatura, por su carácter lúdico, dinámico y vivencial, a diferencia del resto, moviliza la energía corporal y posibilita que afloren los conflictos internos de las personas.

...en educación física afloran los sentimientos y las emociones y..., los conflictos internos afloran, hay veces que, en estas edades, no saben diferenciar y se pasan del juego a hacer agresión real, si se les avisa, se retiran un poco del juego y se tranquilizan. Pero en educación física, hay mucho contacto físico y ahí, el que tiene dificultades para relacionarse o para saber perder o para saber aceptar las reglas de un juego... Y, claro, la educación física es un espacio ideal, entre comillas, para que surjan los conflictos, pero también es un espacio ideal para que se den cuenta de qué es lo que pasa y lo que no pasa (C1_EP2).

En esta misma línea, un profesor añade que en las actividades deportivas el manejo de situaciones de impotencia y frustración resulta complicado, lo que se traduce en una mayor presencia de peleas y conflictos.

...en educación física se dan más conflictos que en otras asignaturas porque hay más contacto físico (...) cuando realizan un deporte están en tensión y cuando se ven impotentes, no saben gestionar la tensión y descargan utilizando las manos... en vez de solucionarlo pegándose les tenemos que preparar para la sociedad (...) tendrán que aprender a solucionarlo hablando (C2_EP).

Espacios de competición: situaciones competitivas de ganary perder

El deseo de ganar es el reflejo de la sociedad competitiva en la que vivimos, que lleva a los niños a enfadarse fácilmente, lo que da lugar a numerosos conflictos. Las voces indican que esta competitividad abarca todos los ámbitos de la vida del escolar, si bien, queda amplificada en los juegos competitivos que tienen lugar durante el recreo, en los entrenamientos y en las clases de educación física.

...los chiquillos compiten para sacar la mejor nota, para hacer los mayores abdominales, para que su grupo sea el mejor y, en algún momento, ponen alguna zancadilla que no se vea o un empujón y ya hay conflicto... Por ejemplo, en el patio... y en educación física, se pican mucho, con juegos competitivos... Porque este es mi grupo y yo tengo que ser el mejor y te tengo que ganar a ti... Yo creo que ahí, sí hay conflictos y en el patio jugando también... (C3_EV).

El alumnado coincide en situar una de las causas en la poca claridad con la que el profesorado explica las normas de juego y los límites de lo permitido y no permitido. 
En gimnasia, cuando hacemos juegos competitivos y hacemos grupos, hay piques y los alumnos se quejan... y después hay conflictos... y es que en las actividades competitivas es muy importante que se planteen bien...que esté todo muy clarito para todos, porque se puede liar buena si el tema no se plantea bien... (C2_RA)

Otro de los detonantes identificados por profesorado y alumnado es la actitud chulesca y burlona que adoptan los que ganan contra los que pierden.

... Cuando en educación física se hacen actividades donde hay un ganador y un perdedor, suele haber más riñas. Porque igual imagínate un chico que te ha ganado una partida de pelota o algo y empieza a burlarse, tú te lo tomas así, mal y empieza a chillarte en tu oreja... y eso molesta... siempre que hacemos actividades de ganar y perder, hay piques... (C1_RA).

Un tercer detonante mencionado por el profesorado y el alumnado son las actitudes irrespetuosas y poco empáticas que adoptan algunos escolares hacia su equipo al insultarles y recriminarles por su falta de habilidad.

... Esta semana una alumna se ha quejado diciendo... que le han insultado porque le han metido gol y han perdido por su culpa... Ahí se insultan. Y dicen cosas como 'corre que eres una vaga o una gorda'... yo les veo darse unos berrinches, echándose las culpas el uno al otro porque te han metido el gol... (C2_EP).

\section{Discusión de resultados}

El objetivo de esta investigación era el de analizar el significado que escolares, familiares, voluntariado y profesorado atribuyen al término conflicto e identificar los contextos a los que asocian su aparición. Al definir lo que entienden por conflicto, todas las personas lo hacen desde la perspectiva de naturalidad e inevitabilidad del mismo (Jares, 2001; Molina-Luque, 2005; París-Albert, 2005; Vinyamata, 2003). Para expresar lo que entienden por conflicto, recurren a la situación conflictiva o manifestación observable, lo cual coincide con la tendencia más extendida (Cascón-Soriano, 2000; Jares, 2001). Las personas adultas se refieren de forma reiterada a las dificultades de niños para gestionar sus enfados de forma no violenta, lo que lleva a plantear la necesidad de prestar atención a la educación emocional, y al trabajo de autoconocimiento que permite comprender y manejar conflictos tanto intrapersonales como interpersonales e intragrupales para posibilitar la mejora de la convivencia (Colectivo
Amani, Aguilera-Reija, Gómez-Lara, Morollón-Pardo \& De Vicente-Abad, 2009; Molina-Luque, 2005; Naranjo, 2014).

La expresión pique, con evidente carga negativa, es la más utilizada por los niños y las niñas para explicar lo que entienden por conflicto. Entre adultos, los términos utilizados son discusiones, riñas, peleas, insultos... Los menores ponen de manifiesto cierto predominio de la percepción negativa del conflicto sobre la percepción positiva (Jares, 2001; Pineda-Alfonso, 2011). No obstante, entre las personas adultas, la puerta está abierta a hacer del conflicto una herramienta pedagógica. Este reconocimiento lo relacionamos con el recorrido realizado como CCAA que se orienta a la construcción de la convivencia educativa inclusiva (Leiva-Olivencia, 2009; Leiva-Olivencia \& Escarbajal-Frutos, 2011).

En este caso, la percepción del alumnado sobre las causas está vinculada al género y al clima de enfrentamiento en el que dicen vivir. Este pique se hace claramente visible en los juegos competitivos en los que algunos chicos se sienten superiores a las chicas, se burlan de ellas y las humillan, al adoptar actitudes machistas que, de acuerdo con Rosa Cobo-Bedia (2006), son el origen de muchos de los conflictos de la sociedad y de la escuela. Estas actitudes machistas demandan una intervención directa por parte de la figura de autoridad (Ruiz, Riuró \& Tesouro, 2015). La percepción de las personas adultas sobre las causas de los conflictos tiene que ver con la competitividad y el deseo de sobresalir, ganar, ser el mejor. Este deseo está fuertemente enraizado en la sociedad patriarcal (Naranjo, 2014; Ortí-Ferreres, 2003).

En los centros investigados, los resultados dejan ver la ausencia de un clima de conflicto, ya sea por razones disciplinarias relacionadas con la ruptura del orden en las aulas, o por diferencias culturales o raciales, lo que en principio lleva a afirmar que el clima de los centros del modelo comunitario no cabría catalogarlo como de "abiertamente conflictivo", como expresan José Ramón Flecha-García y Carmen García-Yeste (2007), si bien esto no quiere decir que sean centros libres de conflicto. Los resultados nos remiten a lugares de la escuela en los que el conflicto está presente, entre los que se destacan el patio y las clases de educación física. Nos preguntamos si esto no se deberá a que el resto del tiempo y de las asignaturas el alumnado está tan centrado en lo mental que cuando llega a un espacio abierto, de libertad, surge el conflicto.

El patio es señalado como el lugar de la escuela en el que se producen más conflictos - lo que coincide con lo descubierto por Rosario Ruiz, María Riuró y Montse Tesouro (2015)—, debido a una menor vigilancia y a la necesidad de jugar y descargar 
tensiones acumuladas. Esto lleva a reflexionar sobre la conveniencia de introducir cambios metodológicos orientados a facilitar la autorregulación emocional, y la responsabilidad personal y social que plantea Don Hellison (2010). Estos cambios deberán prestar más atención a la necesidad de que niños y niñas experimenten con su cuerpo para aprender a escucharlo, respetarlo y cuidarlo, tanto en el aula como fuera de ella (Martínez-Guzmán, 2005; Naranjo, 2014).

La asignatura de educación física es percibida como la disciplina curricular en la que se producen más conflictos al ser la única en la que el alumnado se mueve en libertad (Ortí-Ferreres, 2003). Esto nos lleva a plantear la necesidad de utilizar el escenario privilegiado que ofrece esta asignatura para que cada escolar contacte con su espontaneidad, impulsividad, agresividad, y pueda aprender a sentir, acompañar y expresar la impotencia, la frustración, el enfado y toda la amplia gama de emociones y sentimientos que acompañan las situaciones de enfrentamiento y conflicto (Macazaga-López, Rekalde-Rodríguez \& Vizcarra-Morales, 2013; Vizcarra-Morales, Macazaga-López \& RekaldeRodríguez, 2013, 2016).

Las personas adultas han focalizado el protagonismo en los escolares y ellas quedan fuera de toda situación conflictiva. Este olvido remite a la necesidad de realizar un profundo trabajo de autoconocimiento, porque solo a partir del reconocimiento y la aceptación de los propios conflictos se estará en disposición de reconocer y comprender los de las otras personas (Naranjo, 2014).

Hay que destacar las limitaciones derivadas de un diseño que se ha centrado en indagar en las ideas y percepciones de los participantes sobre el conflicto, como paso previo a la realización de estudios posteriores focalizados en la gestión de los conflictos.

\section{Sobre las autoras}

María Teresa Vizcarra-Morales es profesora doctora titular de la Facultad de Educación de la Universidad del País Vasco/Euskal Herriko Unibertsitatea, UPV/EHU. Su investigación se desarrolla en el ámbito del deporte escolar, la formación del profesorado y la educación emocional.

Itziar Rekalde-Rodríguez es doctora por la Universidad de Salamanca y profesora del departamento de Didáctica y Organización Escolar en la Universidad del País Vasco/Euskal Herriko Unibertsitatea, UPV/EHU. Áreas de experticia: formación del profesorado e innovación educativa.

Ana María Macazaga-López es profesora doctora titular de la Facultad de Educación de la Universidad del País Vasco/ Euskal Herriko Unibertsitatea, UPV/EHU. Su investigación se desarrolla en el ámbito del deporte escolar, la formación del profesorado y la educación emocional.

\section{Referencias}

Antolín de la Fuente, Ángel; Martín-Pérez, Gonzalo \& Barba-Martín, José J. (2012). El aprendizaje cooperativo para la mejora de la socialización y la educación a través del conflicto. La Peonza: Revista de Educación Física para la Paz, 7, 3-11. Disponible en: https://dialnet.unirioja.es/descar ga/articulo/3907247.pdf

Blaya, Catherine \& Debarbieux, Éric (2011). La violencia en los colegios de enseñanza primaria: ¿cómo están los alumnos franceses? magis, Revista Internacional de Investigación en Educación, 4 (8) Edición especial La violencia en las escuelas, 339-356. Disponible en: http://revistas.jave riana.edu.co/index.php/MAGIS/article/down load/3568/2683

Bolívar-Botía, Antonio (2000). Los centros escolares como comunidades. Revisando la colegialidad. Revista Española de Pedagogía, 216 (58), 253274. Disponible en: https://revistadepedagogia. org/wp-content/uploads/2007/06/216-04.pdf

Bonino, Silvia (1987). Bambini e non violenza. Torino: Edizioni Gruppo Abele.

Caba-Collado, María Ángeles de la (1997). La disciplina como planteamiento de educación sociopersonal: pautas de orientación. Revista Española de Orientación y Psicopedagogía, 8 (13), 15-27.

Cascón-Soriano, Paco (2000). ¿Qué es bueno saber sobre el conflicto? Cuadernos de Pedagogía, 287, 57-60.

Cobo-Bedia, Rosa (2006). Ellas y nosotras en el diálogo intercultural. En Cobo-Bedia, Rosa (ed.). Interculturalidad, feminismo y educación. Madrid: Los Libros de la Catarata. Disponible en: http:// www.ciudaddemujeres.com/articulos/Ellas-ynosotras-en-el-dialogo

Colectivo Amani; Aguilera-Reija, Beatriz; Gómez-Lara, Juan; Morollón-Pardo, Mar \& Vicente-Abad, Juan de (2009). Educación intercultural. Análisis y resolución de conflictos. Madrid: Los Libros de la Catarata.

Denzin, Norman K. \& Lincoln, Yvonna S. (2015). Manual de investigación cualitativa. Métodos de recolección y análisis de datos. Barcelona: Gedisa.

Díaz-Aguado, María José (2006). Educación intercultural y aprendizaje cooperativo. Madrid: Pirámide.

Elboj-Saso, Carmen (2011). Socialización preventiva de la violencia de género desde los centros educativos. Organización y gestión educativa: Revista del Fórum Europeo de Administradores de la Educación, 19 (3), 19-23.

Elboj-Saso, Carmen \& Niemelä, Reko (2010). Sub-Communities of Mutual Learners in the Classroom: The Case of Interactive Groups. Revista de 
Psicodidáctica, 15 (2), 177-189. doi: 10.1387/RevPsicodidact.810. Disponible en: http://www.redalyc.org/html/175/17517246003/

Elliott, John (2010). Cambio educativo desde la investigación-acción. Madrid: Morata.

Flecha-Fernández de Sanmamed, Ainhoa M.; García, Rocío; Gómez, Aitor \& Latorre, Antonio (2009). Participación en escuelas de éxito: una investigación comunicativa del proyecto Includ-ed. Revista Cultura y Educación, 21 (2), 183-196. Disponible en: http://www.reus.cat/sites/ reus/files/05_08_participacion_escuelas_de_exito_included.pdf

Flecha-Fernández de Sanmamed, Ainhoa M.; Melgar, Patricia; Oliver, Esther \& Pulido, Cristina (2010). Socialización preventiva en las comunidades de aprendizaje. Revista Interuniversitaria de Formación del profesorado, 67 (24,1), 89-100. Disponible en: https://dialnet.unirioja.es/ descarga/articulo/3148952.pdf

Flecha-García, José Ramón \& García-Yeste, Carmen (2007). Prevención de conflictos en las comunidades de aprendizaje. Idea La Mancha: Revista de Educación de Castilla La Mancha, 4, 72-76. Disponible en: http://redined.mecd.gob.es/xmlui/bitstream/hand le/11162/93546/00620073000915.pdf?sequence =1

Flick, Uwe (2014). El diseño de la investigación cualitativa. Madrid: Morata. Gómez, Aitor; Puigvert, Lidia \& Flecha, Ramón (2011). Critical Communicative Methodology: Informing Real Social Transformation through Research. Qualitative Inquiry, 17 (3), 235-245. Disponible en: http://bu rawoy.berkeley.edu/Public\%20Sociology,\%20Live/Flecha.Critical\%20 Communicative\%20Methodology.pdf

Gómez, Jesús; Latorre, Antonio; Sánchez, Montse \& Flecha, Ramón (2006). Metodología comunicativa crítica. Barcelona: El Roure.

Hellison, Don (2010). Teaching Personal and Social Responsibility through Physical Activity. Champaign, Illinois: Human Kinetics.

Jares, Xesús R. (2001). Educación y conflicto: guía de educación para la convivencia. Madrid: Editorial Popular.

Lavega, Pere; Filella, Gemma; Lagardera, Francisco; Mateu, Mercé \& Ochoa, Jordi (2013). Juegos motores y emociones. Cultura y Educación, 25 (3), 347-360. DOI:10.1174/113564013807749731

Leiva-Olivencia, Juan J. (2009). El profesorado ante la concepción de los conflictos en contextos de educación intercultural. Aula Abierta, 37 (2), 93-110. Disponible en: https://dialnet.unirioja.es/servlet/ articulo?codigo $=3088579$

Leiva-Olivencia, Juan J. \& Escarbajal-Frutos, Andrés (2011). La participación de las familias inmigrantes como fundamento pedagógico en la construcción de la interculturalidad en la escuela. Educatio Siglo XXI, 29 (2), 389-416. Disponible en: http://revistas.um.es/educatio/ article/view/133121

Llamas-Toledano, Josué (2003). Orientaciones para afrontar y educar en el conflicto desde la educación física. Tándem: Didáctica de la Educación Física, 13, 51-62.

Lowen, Alexander \& Lowen, Leslie (2005). Ejercicios de bioenergética. Barcelona: Sirio.

Macazaga-López, Ana María; Rekalde-Rodríguez, Itziar \& Vizcarra-Morales, María Teresa (2013). ¿Cómo encauzar la agresividad? Una propuesta de intervención a través de juegos y deporte. Revista Española de Pedagogía, 71-255, 263-276. Disponible en: https://reunir.unir. net/handle/123456789/3766

Martín-Hocajo, Montse \& Ríos-González, Oriol (2014). Prevención y resolución del conflicto en educación física desde la perspectiva del 
alumnado. Retos: nuevas tendencias en educación física, deporte y recreación, 25, 162-167.

Martínez-Guzmán, Vicent (2005). Podemos hacer las paces. Reflexiones éticas tras el 11-S y el 11-M. Bilbao: Desclée de Brouwer, DDB.

Molina-Luque, Fidel (2005). Nuevos conflictos sociales y su presencia educativa. Análisis sociológico y reflexiones para la intervención. Cultura y Educación, 17 (3), 213-223.

Monjas-Casares, María Inés \& González-Moreno, Balbina de la Paz (2001). Las habilidades sociales en el currículo. Madrid: Ministerio de Educación Cultura y Deporte, Centro de Investigación y Documentación Educativa.

Monzonís-Martínez, Núria \& Capllonch-Bujosa, Marta (2014). La educación física en la consecución de la competencia social y ciudadana. Retos: Nuevas Tendencias en Educación Física, Deporte y Recreación, 25, 180-185. Disponible en: http:// www.redalyc.org/pdf/3457/345732291035.pdf

Naranjo, Claudio (2014). Cambiar la educación para cambiar el mundo. Barcelona: La Llave.

Ortega, Pedro; Mínguez, Ramón \& Saura, Pilar (2003). Conflicto en las aulas. Barcelona: Graó.

Ortí-Ferreres, Joan (2003). La resolución de conflictos en la educación física. Tándem: Didáctica de la Educación Física, 12, 40-50.

Palomero-Fernández, Pablo (2010). Desarrollo de la competencia social y emocional del profesorado: aproximación humanista desde la psicología humanista. Revista Electrónica de Formación del Profesorado, 29 (12), 145-153. Disponible en: https://dialnet.unirioja.es/descarga/articu lo/3039102.pdf

París-Albert, Sonia (2005). La transformación de los conflictos desde la filosofía para la paz. Tesis doctoral Universitat Jaume I, Barcelona. Disponible en: http://www.tdx.cat/bitstream/hand le/10803/10456/paris.pdf

París-Albert, Sonia \& Martínez-Guzmán, Vicent (2010). Interculturalidad y conflicto. Una perspectiva desde la filosofía de la paz. Investigaciones Fenomenológicas: Anuario de la Sociedad Española de Fenomenología, 7 (5), 85-97. Disponible en: http://www2.uned.es/dpto fim/InvFen/In vFen07/pdf/06_PARIS_et_al.pdf

Pérez-Galán, Rafael (2009). Los nuevos retos del aprendizaje social en niños con necesidades educativas especiales: el aprendizaje en comunidad o la comunidad de aprendizaje en el aula. Revista de Educación, 348, 443-464. Disponible en: http://www.revistaeducacion.mec.es/re348/ re348 19.pdf

Perrenoud, Philippe (2004). Desarrollar la práctica reflexiva en el oficio de enseñar. Barcelona:
Graó. Disponible en: https://coleccion.siae ducacion.org/sites/default/files/files/6_perre noud_philippe_2007desarrollar_la_practica_ reflexiva.pdf

Pineda-Alfonso, José Antonio (2011). Las concepciones de los alumnos sobre el conflicto y la convivencia. Un estudio con alumnado de $4^{\circ}$ de ESO. Investigación en la Escuela, 75, 35-47. Disponible en: https://idus.us.es/xmlui/handle/11441/25045

Ruiz, Rosario; Riuró, María \& Tesouro, Montse (2015). Estudio del bullying en el ciclo superior de primaria. Educación XX1, 18 (1), 345-368. DOI: 10.5944/educXX1.18.1.12384. Disponible en: http://revistas.uned.es/index.php/educacionXX1/ article/view/12384/12227

Sáez de Ocáriz, Unai; Lavega, Pere \& March, Jaume (2013). El profesorado ante los conflictos en la educación física. El caso de los juegos de oposición en primaria. Revista Electrónica Interuniversitaria de Formación del Profesorado, 16 (1), 163176. Disponible en: http://revistas.um.es/reifop/ article/viewFile/180101/151111

Salinas-Salazar, Marta Lorena; Posada-Giraldo, Diana María \& Isaza-Mesa, Luz Stella (2002). A propósito del conflicto escolar. Revista Educación y Pedagogía, 14 (34), 243-273. Disponible en: http:// aprendeenlinea.udea.edu.co/revistas/index.php/ revistaeyp/article/view/325110/20782596

Trianes-Torres, María Victoria (2000). La violencia en contextos escolares. Málaga: Aljibe.

Valles-Martínez, Miguel S. (2003). Técnicas cualitativas de investigación social. Madrid: Síntesis Sociología.

Vargas, Julio \& Flecha, Ramón (2000). El aprendizaje dialógico como 'experto' en resolución de conflictos. Contextos Educativos: Revista de Educación, 3, 81-88. Disponible en: https://dialnet. unirioja.es/descarga/articulo/201068.pdf

Vinyamata, Eduard (2003). Comprender el conflicto y actuar educativamente. En Eduard Vinyamata (coord.). Aprender del conflicto. Conflictología y educación, 9-28. Barcelona: Graó.

Vizcarra-Morales, María Teresa; Macazaga-López, Ana María \& Rekalde-Rodríguez, Itziar (2013). El proceso de acompañamiento en la construcción participativa de una normativa. Revista Española de Orientación y Psicopedagogía, 24 (1), 110-120. Disponible en: http://revistas.uned.es/ index.php/reop/article/view/11274/10796

Vizcarra-Morales, María Teresa; Macazaga-López, Ana María \& Rekalde-Rodríguez, Itziar (2016). How Conflicts Are Resolved in Three Learning Communities. Revista de Psicodidáctica, 21 (2), 281-301. Disponible en: http://www.ehu.eus/ ojs/index.php/psicodidactica/article/viewFile/ 14772/14204 